\title{
Testing annual asthma reviews for those who fail to attend: proof-of-concept study
}

By Miriam Craske, David Wright, Jeanette Blacklock, Heather Matthews, Tony Dean, Tania Farrow , Clare Daly, Paul Duell

\begin{abstract}
Introduction: Suboptimal asthma care is related to increased morbidity and mortality. As a result, GP surgeries provide annual reviews for people with asthma. A high proportion of asthma patients do not attend their review but still collect asthma treatment from their community pharmacy. The aim of this proof-of-concept study was to evaluate the provision of patient non-attendance lists to community pharmacies who subsequently offered the review
\end{abstract}

Method: Five GP surgeries and ten community pharmacies were recruited in the east of England. Non-attender details were provided to community pharmacies from GP surgeries directly over a six-month period. Asthma reviews, funded by the medicines use review scheme, were delivered using standardised methodological approaches and electronic recording systems. Relevant routinely collected data were obtained before and after service provision. Stakeholder meetings were held to obtain feedback after service completion.

Results: A total of 27 patients received the service, with data collected on 26 patients. High levels of satisfaction with the service were identified. Pharmacist training, pharmacy accessibility and pharmacist competence were seen as service enablers. The pharmacy consultation room, GP surgery organisation and different IT systems were seen as barriers. A high level of satisfaction was identified, with the recommendation that the service should be offered to all patients with asthma irrespective of attendance.

Discussion and conclusion: This service model, which involved integrated working between GPs and community pharmacies and enabled asthma review non-attenders to be targeted, is in line with the recommendations of the Murray Review. Stakeholders recommended that community pharmacists should provide yearly asthma reviews and that these should be performed in close collaboration with GP surgeries.

Keywords: Asthma; asthma review; community pharmacy; GP referral; medicines use review; MUR; pharmacists. 


\section{Key points}

The community pharmacy asthma review service (CPARS) widens patient choice

- and enables patients who usually do not attend at the GP surgery to receive an asthma review.

Patients, pharmacists and GP surgery staff all reported a high level of satisfaction

- with CPARS.

- Pharmacists need to undertake additional training to enable them to provide asthma reviews at a standardised level.

- CPARS encourages GP surgeries and community pharmacies to work together.

\section{Introduction}

Around 5.4 million people in the UK receive treatment for asthma, costing the NHS more than $£ 1$ bn per year and resulting in 1.1 million lost working days ${ }^{[1]}$. In addition, $70 \%$ of asthmarelated accident and emergency (A\&E) visits and hospital admissions and $90 \%$ of asthmarelated deaths are believed to be preventable with appropriate treatment and monitoring $[2]$. The 2015 National Review of Asthma Deaths (NRAD) found that the number of deaths per year caused by asthma remained at 1,200[3]. Patients from lower socioeconomic groups are also less likely to have their asthma under control than patients from higher socioeconomic groups and, consequently, asthma has more of a negative effect on their usual daily activities ${ }^{[4]}$. 
The National Institute for Health and Care Excellence recommends that all patients with asthma receive a structured review at least annually ${ }^{[5]}$. The review should monitor how well the patient's condition is controlled, and ensure that they continue to be prescribed the most effective treatment and are supported to use their therapy appropriately. The review should include provision of a written personalised action plan, an asthma control assessment and specific training in inhaler technique $\frac{[5]}{}$.

However, around 30\% of asthma patients fail to attend their asthma review for many reasons, including not believing their asthma warranted a review and forgetting to attend $[6]$. Prescriptions for asthma treatment continue to be collected through community pharmacies and this provides an opportunity for the asthma review to be offered when patients collect their asthma therapy. Offering additional services while patients access another health service is known to result in greater service uptake than requests for patients to receive a service via an additional appointment ${ }^{[7]}$. With patients reporting a preference for services that have been authorised by their $\mathrm{GP}^{[8]}$, offering patients their asthma review at the point of prescription collection in the knowledge that this has been recommended by their GP should encourage service uptake.

The use of community pharmacists to successfully identify and manage asthma has been widely reported in international literature ${ }^{[9],[10]}$. In the UK, several studies have demonstrated wide acceptance for, and the potential value of, community pharmacists providing medicinesrelated asthma and respiratory services to patients $\frac{[11],[12],[13],[14]}{}$. However, none of the reported services have been delivered in close cooperation with GP surgeries, whereby patients who fail to attend their yearly review are approached by their community pharmacist ${ }^{[15]}$.

Community pharmacists in the UK are currently remunerated to provide advice on medicines use to patients with asthma through the national medicines use review (MUR) scheme; this model has been used in both the UK and Italy to enhance the care of patients with asthma ${ }^{[11],[16]}$. One large-scale UK study identified that the service could be enhanced if GPs were encouraged to refer patients to the service ${ }^{[11]}$. Latif et al. identified that the lack of collaboration between community pharmacists and GPs surrounding the delivery of MURs is a lost opportunity to enhance patient care ${ }^{[17]}$. The consequence of community pharmacists offering the service to patients without referral from their GP is the potential for duplication of effort. Within the main UK asthma MUR service evaluation, 70\% of reviews provided by community pharmacists were for patients who had already received an asthma review from their GP surgery in the previous year $[11]$.

The recent review of clinical pharmacy services in the UK recommends that the existing MUR element of the pharmacy contract should be redesigned to support the management of longterm conditions; this should be better integrated with GP surgeries to ensure that patients who are most in need are identified and that services are more efficiently delivered ${ }^{[18]}$.

Concerns regarding large numbers of unnecessary asthma-related hospitalisations to James Paget University Hospital in Great Yarmouth, UK, led the hospital, Great Yarmouth and Waveney Clinical Commissioning Group, the local pharmaceutical committees (LPCs), the Centre for Pharmacy Postgraduate Education and the University of East Anglia to collectively develop a community pharmacy asthma review service (CPARS) for GP surgery nonattenders. This was delivered through the existing MUR service, with additional funding provided to cover the costs of involvement and data collection.

The widespread location of community pharmacies, combined with their opening hours being outside usual office hours, was seen as an opportunity to reach deprived areas and encourage attendance for those employed in roles with limited flexibility. Consequently, increasing access to asthma reviews through community pharmacies has the additional potential of reducing the known health inequalities in asthma management ${ }^{[4]}$. The aim of this proof-ofconcept study was to evaluate the service for acceptability and future refinement. 


\section{Method}

The University of East Anglia Faculty of Medicine and Health Ethics Committee deemed the project to be a service evaluation.

A total of five GP surgeries and their ten associated community pharmacies were recruited within the Great Yarmouth and Lowestoft area. Patients who had not received an asthma review in the previous 12 months were identified and their community pharmacist informed of the need to review the patient, with their consent, when they presented for a prescription. GP surgeries decided which patient names were provided to the pharmacies; no specific exclusion criteria were set.

\section{Pharmacist training}

Fifteen pharmacists from ten community pharmacies attended six hours of training, divided over two evenings. Each pharmacy was also provided with a support pack to aid the review process. A summary of the training and support pack is provided in Box 1.

Box 1: Pharmacist training content and support pack

\section{Pharmacist training}

- Project introduction;

- Inhaler technique and asthma management;

- Workshop session using In-Check ${ }^{\mathrm{TM}}$ (Alliance) device, peak flow meters and placebo inhalers;

- Expert overview on asthma service delivery;

- Role play with actors to undertake asthma review with formative feedback;

- Clinical case studies;

- The SIMPLE (stop smoking, inhaler technique, monitoring, pharmacotherapy, lifestyle and education) approach to the management of asthma $\stackrel{[23]}{ }$.

\section{Support pack content}

- In-Check Dial and disposable (white) mouthpieces;

- Airzone ${ }^{\mathrm{TM}}$ (Alliance) standard peak flow meter and disposable mouthpieces (red);

- Space chamber compact spacer device;

- Placebo inhalers (relievers and preventers):

-Ellipta® (GlaxoSmithKline);

- Respimat@ (Boehringer);

-Nexthaler® (Chiesi);

- Easyhaler® (Orion);

— DuoResp ${ }^{\circledR}$ Spiromax ${ }^{\circledR}$ (Teva Pharma);

- Metered dose inhaler (Fostair ${ }^{\circledR}\left[\right.$ Chiesi] / Ventolin ${ }^{\mathrm{TM}}\left[\right.$ GlaxoSmithKline]/ Clenil ${ }^{\circledR}$

[Chiesi]);

— Turbohaler® (AstraZeneca);

— Genuair® (AstraZeneca).

Training devices: 
-Ellipta training device;

-Turbohaler training whistles.

- Asthma support pack (peak flow diary and blank asthma management plan in A5 wallet);

- Steroid cards for patients receiving high dose inhaled corticosteroids;

- Summary of British Thoracic Society treatment guidelines with current clinical commissioning group prescribing guidelines;

- Information for patients: Staying in control of asthma.

\section{Service design}

From March 2017, the GP surgeries identified adult patients who had failed to attend their annual asthma review. Their names were provided to the participating community pharmacies and these patients were offered an annual review when they presented for their prescription. The review was arranged for a mutually convenient time. Figure 1 provides a summary of the service.

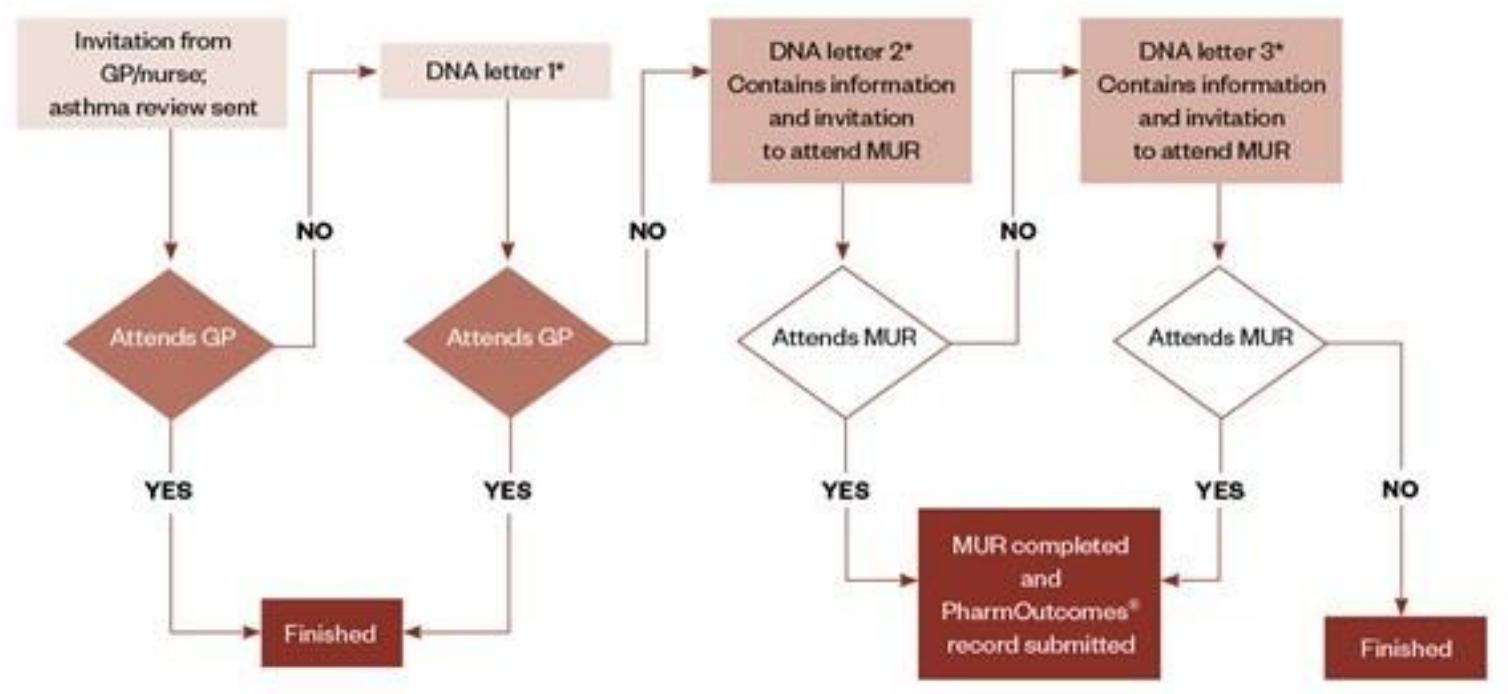

DNa = did not attend, MUP = mediches use noview

-Each Defa lotter is sent inmorchly intervals Thio is a throe-month prooss.

Figure 1: Summary of service design

From March 2017, the GP surgeries identified adult patients who had failed to attend their annual asthma review. These patients were offered another review when they presented for their prescription, arranged for a mutually convenient time

\section{Intervention delivery}

The design and content of the asthma review was agreed by the lead respiratory nurse from James Paget University Hospital and the chief officer of Suffolk LPC, and this was used to develop the PharmOutcomes ${ }^{\circledR}$ (Pinnacle Health Partnership) - a national web-based community pharmacy intervention recording system. In addition to the standard MUR questions, the information that was assessed/discussed and subsequently collected using PharmOutcomes is provided in Box 2. 
The PharmOutcomes system enables an email confirmation to be sent to the patient's GP surgery when they have had an asthma review. This information allows the GP surgery to claim their quality and outcomes framework (QOF) points 19$]$.

Pharmacy contractors received their MUR fee for each asthma review delivered plus a fee of $£ 15$ for completing PharmOutcomes during the process. They were paid $£ 175$ for each pharmacist that attended both training sessions, which equated to six hours of learning.

\section{Box 2: Asthma review content recorded via PharmOutcomes ${ }^{\circledR}$}

- Asthma Control Test $\frac{[24]}{}$;

- Number of asthma-related attendances at accident and emergency in the past 12 months;

- Smoking status assessment and offer of cessation service where appropriate;

- Asthma trigger factors and plans for management;

- Presence of symptoms of uncontrolled rhinitis;

- Flu vaccination status and offer of flu vaccination where appropriate;

- Adherence to current prescribed asthma medication;

- Use of spacer for metered dose device;

- Assessment of inhaler technique using in-check dial and associated intervention(s);

- Measurement of peak expiratory flow rate and access to a peak flow meter;

- Dose of inhaled corticosteroid and whether step-down therapy could be considered;

- Need for a steroid card;

- Identification of presence of an asthma management plan and associated intervention.

\section{Data collection}

The following quantitative and qualitative data were collected:

- Content of pharmacist reviews;

- Patient satisfaction;

- Routine asthma-related data;

- Stakeholder feedback;

- Pharmacist review content collected via PharmOutcomes (summarised in Box 2).

Patients were asked for their preference on the mode of delivery of a service evaluation patient satisfaction questionnaire (i.e. postal or online) and sent the survey one month after service delivery. Along with an open question to allow for qualitative comments about the service, the questionnaire included questions regarding:

- Their demographics;

- The community pharmacy where the service was delivered;

- The asthma control test;

- Their satisfaction with the consultation;

- Their willingness to receive the asthma review via this route in the future;

- Whether they would recommend the review to others;

- What they had changed as a result of the service.

The following data were collated by GP surgeries and provided in an anonymous format for evaluation purposes, and included all patients who received the asthma service through their community pharmacy for three months before and three months after service delivery: 
- Unplanned asthma-related hospitalisations;

- Unplanned asthma-related GP visits;

- Preventer inhaler use;

- Reliever inhaler use.

Patients, GP surgery staff and pharmacists involved in the delivery of the service were invited to stakeholder meetings with the project research associate (non-pharmacist) to obtain feedback on the service. Two patient and public involvement members of the management team supported the patient stakeholder meeting and were not recorded as participants. All of the patient and pharmacist participants were independent of the project team. Two GP surgery staff who attended were seconded to the project team after the original grant had been submitted and were employed by the GP surgery that held the project grant.

Only one patient who completed the survey offered to attend a stakeholder feedback meeting. Therefore, the main GP surgery wrote to their 'usual' non-attenders to invite them in to identify why they do not attend and their views on offering the service through community pharmacy. Meetings were arranged at suitable times and locations, with food and remuneration for attendance provided.

The questions asked included:

- What were your thoughts about community pharmacists providing asthma reviews?;

- Which elements helped/hindered the delivery of the service?;

- What was the effect of the service on your role?;

- How could the service be upscaled/expanded?;

- What are your thoughts on pharmacist training and support? (Pharmacist stakeholder meeting only);

- How could the GP practice help you to attend your annual asthma review? (Patient stakeholder meeting only);

- What barriers prevented you from attending your annual asthma review appointment? (Patient stakeholder meeting only).

Consent to record the meetings was obtained and notes were taken by a second member of the team. Two patient and public involvement panel group members assisted with the patient stakeholder meeting. The recordings were transcribed or listened to in order to check the notes for accuracy. Identified barriers, enablers and solutions were extracted by two members of the management team. Patient and public involvement members of the management team also reviewed the patient stakeholder meeting transcriptions.

\section{Results}

A total of 27 patients received the service from six different community pharmacies.

\section{Pharmacist review content}

Box 3 provides a summary of the reviews and the outcomes arising from them. The community pharmacists adhered to the requirements for service delivery and, in most cases, asthma inhaler technique was addressed during the review.

Box 3: Summary of service delivery using the SIMPLE model

\section{Smoking cessation}

- All patients were asked about smoking status; 
- $11.1 \%$ of service recipients were smokers;

o All smokers were offered and declined smoking cessation.

\section{Inhaler technique}

- All patients had their inhaler technique assessed;

- $64.5 \%$ of patients were identified as having either an inappropriate technique or inhaler;

- All patients with inappropriate inhaler devices were referred back to their GP surgery;

- Counselling addressed inhaler technique in all remaining cases.

\section{Monitoring}

All patients completed the asthma control test;

o $55.6 \%$ of patients had controlled asthma;

- $44.4 \%$ of patients had a peak expiratory flow rate (PEFR) of $80 \%$ or more of their predicted PEFR;

- $40.7 \%$ of patients had a peak flow meter at home to enable self-monitoring.

\section{Pharmacotherapy}

- $18.5 \%$ of patients had more than three short-acting beta agonists (relievers) in six months;

- $7.4 \%$ of patients considered for a reduction in the dose of their inhaled corticosteroid (preventer);

- $11.1 \%$ of patients required a spacer device for their inhaler.

\section{Lifestyle}

All patients were questioned about asthma triggers and plans to manage these triggers.

\section{Education}

- All patients were asked about their asthma management plan;

- $51.9 \%$ of patients were referred to obtain a plan from their GP or asthma nurse.

\section{Patient satisfaction}

In total, seven female patients completed the survey; three were aged 31-50 years, three were aged 51-65 years and one was aged over 65 years. Responses came from patients who had received the service from one of four different community pharmacies.

- Six patients rated their asthma as 'well controlled' and one patient rated their asthma as 'completely controlled';

- All patients agreed that they were satisfied with the information provided during the consultation;

- All patients would have their review at the pharmacy again;

- Six patients would recommend the service to others, while one patient was unsure;

- Three comments were made regarding the unsuitability of the consultation rooms;

- Two patients reported a better inhaler technique;

- One patient reported taking their preventer inhaler more often;

- One patient reported monitoring their condition more carefully. 
Data were collected for 26 patients. There were six recorded unplanned asthma-related visits to the GP from six patients in the three months prior to the service and ten recorded unplanned asthma-related visits to the GP in the three months after, with four visits by one patient. There were no unplanned hospitalisations caused by asthma recorded before or after CPARS.

The use of reliever and preventer inhalers three months before and after the community pharmacy-led review are summarised in Figures 2 and 3.

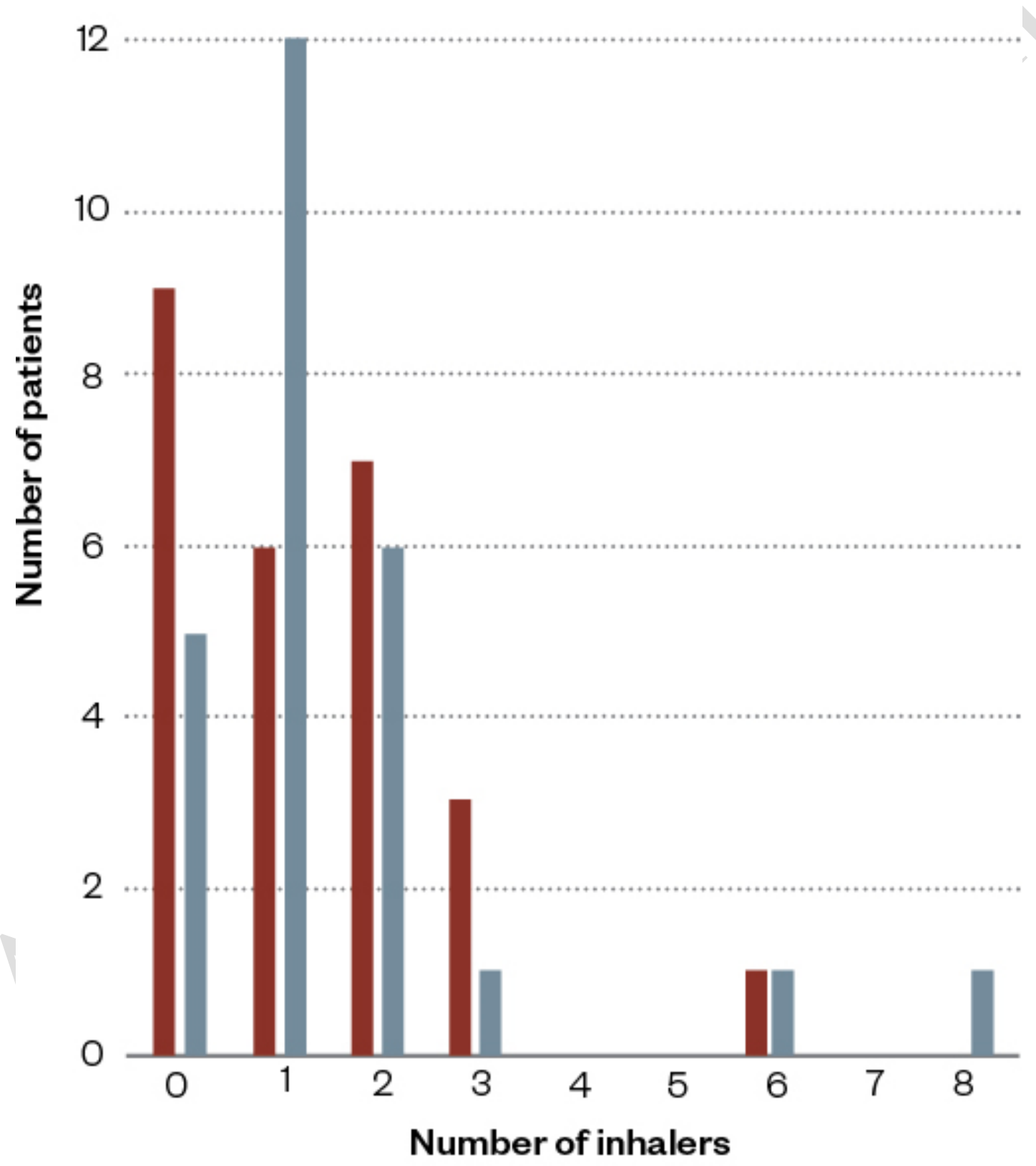

Figure 2: Summary of asthma reliever use three months before and after community pharmacy reviews

Following the review, it was found that some patients were more likely to order at least one reliever inhaler 


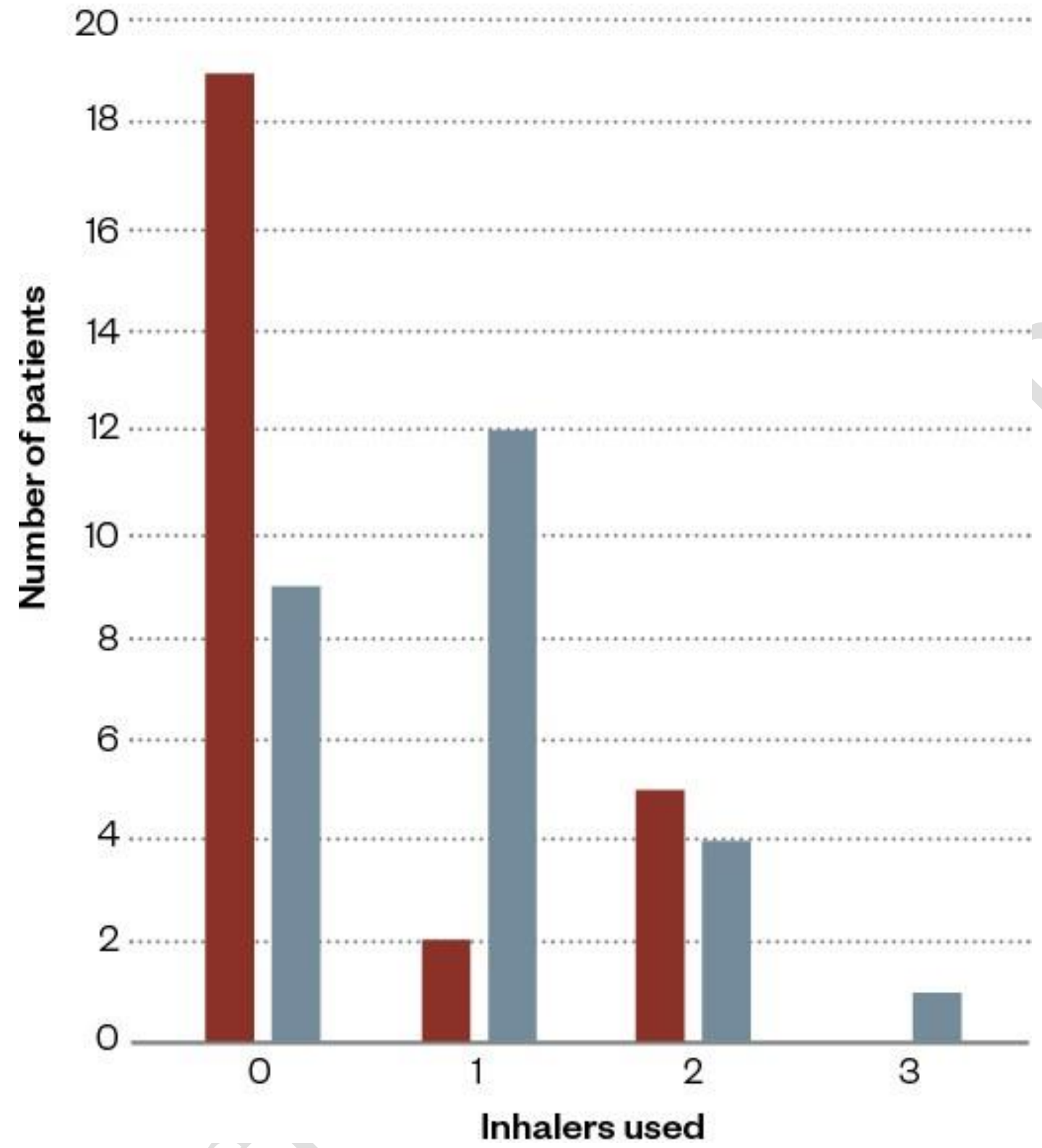

Figure 3: Summary of asthma preventer use three months before and after community pharmacy reviews

More preventer inhalers were ordered three months after intervention, in line with the aim of the review

\section{Stakeholder meetings}

\section{GP surgery staff}

Five medical staff from three different GP surgeries attended. They identified community pharmacists not having access to patient notes as a barrier to effective service delivery, and that this was owing to incompatible IT systems. Enablers for the service were that the service reduces practice nurse time and that patients are not required to make an appointment. 
The GP surgeries recommended that, in order to recruit more patients, all community pharmacies located around the practice should be involved, and that the community pharmacy route for yearly asthma review should be offered to all asthma patients and not limited to nonattenders.

\section{Pharmacists}

Four pharmacists from different community pharmacies attended and an additional pharmacist from another pharmacy was interviewed. Barriers to effective service delivery were identified as the increased time taken to complete reviews and the inability to amend prescriptions themselves. They also identified that non-attenders at GP surgeries are also frequent pharmacy non-attenders (i.e. they use relatives to collect their prescriptions). The elements that enhanced the service were the training that increased self-confidence and the support pack. Improved patient relationships were also seen as encouraging service provision.

The pharmacists recommended that marketing the service should be considered to improve uptake and that the service should be offered to all asthma patients and not just nonattenders. Consideration of how pharmacy technicians could be effectively used within the service model was also recommended.

\section{Patients}

One patient who received the service and four patients who were deemed usual 'nonattenders' attended the meeting The main reason stated for not attending for asthma review was a perceived lack of need. Barriers to using community pharmacies for yearly asthma reviews were lack of privacy, inadequacy and stigma of consultation rooms, lack of rapport with the community pharmacist, lack of service awareness, and that pharmacists are unable to change prescriptions. The perceived competency of community pharmacists, convenience, increase in choice, and ability to ease pressure on GPs were believed to increase service acceptability and adoption.

To improve service uptake through community pharmacies, the patients recommended better consultation rooms, provision of an online appointment system, screening for patients identified most at need through their inhaler consumption, and having compatible GP community pharmacy computer systems.

\section{Discussion}

The driver for this service development was a respiratory nurse specialist, based in secondary care, who had identified a number of preventable asthma-related hospital admissions and wanted to ensure that the NRAD report recommendations were implemented ${ }^{[3]}$. This was particularly important in a known area of deprivation where outcomes related to asthma are known to be worse ${ }^{[4]}$. Through the development of a multidisciplinary team, including all relevant stakeholders, it was possible to secure funding to enable the service to be proof-ofconcept tested and evaluated. Alignment of the team's aims with the recently published Murray Review was used to support the argument for funding the project ${ }^{[18]}$. The project was largely successful owing to the central GP surgery recruiting others to take part and the LPCs aiding identification and recruitment of related community pharmacies. 
Community pharmacists demonstrated that they could provide all elements of the service, as agreed at the outset of the project, therefore giving reassurance regarding service

standardisation. Furthermore, patients who provided feedback on the service (although small in number) were positive regarding their overall experiences. A total of 27 patients were seen who would not normally have attended a review; two-thirds of these patients were assessed as having either an inappropriate inhaler technique or device. Consequently, the patients in need of a review were largely being identified. Half of the patients seen did not have an asthma management plan; this may be owing to their routine non-attendance for asthma review. The time allocated to the review was insufficient to develop an asthma management plan within the pharmacy and, therefore, all were referred back to their GP surgery. Similarly, so were patients who needed a dose reduction or inhaler change.

Community pharmacists reported that the reviews took between 20 minutes and 40 minutes to deliver. As a result of the perceived improvement in relationships with patients, community pharmacists reported patients returning to seek further advice and, in some cases, to have their inhaler technique rechecked. Consequently, the use of the MUR funding model as it currently stands may not be appropriate. The actual cost of service delivery and any associated followup meetings would need to be calculated to ensure that the activity was appropriately remunerated.

The additional cost associated with this workload within the GP surgery would hopefully be justified by improvements in patient quality of life, length of life, reductions in hospitalisation, and unplanned GP visits 20$]$. However, evidence to test this assertion is not available. The service would be more efficiently delivered if the pharmacist could make the changes to the prescription, respond directly to patients requesting large numbers of reliever inhalers or small numbers of preventer inhalers, and if they were remunerated to develop asthma action plans.

Pharmacists also valued the face-to-face training. This not only improved their confidence, but also provided reassurance to the management team regarding the quality of service delivery. The provision of a support pack that included placebo devices, guidelines and an In-Check Dial was also well received because it enabled the pharmacists to effectively assess inhaler technique and respond to any problems they identified. Evaluation of the training identified the need to focus pharmacists' time on active learning activities, and the need for the provision of insight by experts and any associated knowledge to be provided in advance. The training over two evenings, outside normal working hours, was seen as demanding for most pharmacists as they attended after a full day at work. A preference for one day out of work for the training was stated.

The negative patient perceptions associated with a pharmacist consultation room were surprising: these included that the room was used to dispense methadone and discuss personal problems, and as such patients would not want to be seen entering it. Perhaps patient views on this element of pharmacist services should be more robustly sought to identify whether the views identified here are representative and, if so, to identify what needs to change to make them more acceptable. As consultation rooms are more ubiquitously used for provision of services such as influenza vaccinations, the reported stigma associated with their use should diminish.

Perhaps the most positive result was recognition by the GP surgeries that enabling community pharmacists to provide asthma reviews could free up practice nurse time to undertake other activities. They were also supportive of the service being offered through this route for all asthma patients and not just those who do not attend asthma reviews. The pharmacist and patient stakeholder groups independently made the same recommendation. Such a proposal would require close working relationships between the GP surgery and community pharmacies, as well as sharing of records, which aligns with recent recommendations regarding how community pharmacy services should evolve $\frac{[18]}{}$. 
There is also a need to consider the most appropriate funding source. If practice nurse time is freed up, then perhaps the GP surgery should fund the service. Similarly, if the service supports a number of GP surgeries, helps to meet local targets and reduces other NHS costs, the clinical commissioning group may be most appropriate to fund it. Alternatively, the national funding scheme for the MUR could be reconfigured as has been recommended for this purpose ${ }^{[18]}$.

However, the biggest barrier to this service was the creation of non-attender lists; processes within GP surgeries varied significantly from routine list creation within standardised searches to handwritten notes provided by the practice manager. The recent government initiative to put pharmacists in GP surgeries could be used to improve this process $\frac{[21]}{}$.

The regular movement of pharmacists between pharmacies somewhat surprised the pilot study authors, and created unexpected difficulties in ensuring that there was sufficient opportunity for patients from different GP surgeries to attend a community pharmacy for an asthma review. Ultimately, participation of a further GP surgery was lost from the project as a result of this. An additional GP surgery was recruited; however, owing to its late involvement and changes in infrastructure, it failed to deliver any referrals.

\section{Limitations}

The small number of patients seen by the community pharmacists reflects the nature of this group of patients who are, by definition, hard to reach. The reasons for the low service uptake were identified as: intentional non-attendance by patients; patients not visiting community pharmacies because third parties collected their medicines; and community pharmacies who were responsible for significant proportions of participating GP surgery patients not participating in this pilot. In a future service, the inclusion of all community pharmacies and pharmacists around participating GP surgeries would help overcome some of these problems.

This was a small-scale service evaluation of a service with limited patient uptake. As a before and after study, without a control arm, it is not possible to predict what would have happened to these patients if this service had not been provided or how much of the change seen is just owing to the regression to the mean (i.e. the phenomenon that when a variable is found to be extreme at the first measurement, it is likely to be closer to the mean on the second measurement).

Similarly, feedback was obtained from a small proportion of patients and other stakeholders. Ideally, data would have been collected on the number of names provided to the pharmacies to determine the recruitment rate; however, this was not considered when this service was set up. Consequently, it is important that the limited quality of these data and evaluation is recognised.

\section{Conclusion}

This proof-of-concept service evaluation suggests that the asthma service is both feasible and acceptable, and this is part of the process of developing a definitive trial for evaluation of such a complex intervention 22$]$. It also provides some insight into the barriers and enablers to its delivery. For the service to work, pharmacists need to be adequately prepared, the remuneration model needs careful consideration, and GP surgery systems for patient identification need to be organised to enable effective communication. Better integration of record systems would help, as would the pharmacists being able to prescribe themselves. The inclusion of pharmacy technicians within the process also needs to be considered. 
The next step would be to scale up this service and test it across a geographical area where all GP surgeries, practice-based pharmacists and community pharmacists are actively engaged. Ideally, a more formal evaluation could then be undertaken to enable modelling for service effectiveness and cost-effectiveness.

This innovative approach to improving patient understanding and management of their asthma through access to another healthcare professional who is perceived as competent to deliver the service should be considered by commissioners. This is particularly important if there is a desire to meet the NRAD recommendations that were designed to reduce the large number of preventable asthma deaths occurring each year in the $\mathrm{UK}^{[3]}$.

\section{Financial and conflicts of interest disclosure:}

Funding for this project was secured from the Health Foundation's Innovating for Improvement programme. Napp Pharmaceuticals supplied the in-check dials and disposable mouthpieces that pharmacists used during the asthma reviews.

GSK, Boehringer Ingelheim, Chiesi, Orion Pharma, Teva and AstraZeneca all provided placebo devices to be included in the support packs. Teva also provided the 'Let's talk about what asthma means for you' educational material for each pharmacy.

David Wright undertakes consultancy work for large community pharmacy multiples. No other relevant affiliations of financial involvement with any other organisations have been made.

\section{Acknowledgements}

The project was funded by the Health Foundation through its Innovating for Improvement funding scheme.

\section{Reading this article counts towards your CPD}

You can use the following forms to record your learning and action points from this article from Pharmaceutical Journal Publications.

Your CPD module results are stored against your account here at The Pharmaceutical Journal. You must be registered and logged into the site to do this. To review your module results, go to the 'My Account', tab and then 'My CPD'.

Any training, learning or development activities that you undertake for CPD can also be recorded as evidence as part of your RPS Faculty practice-based portfolio when preparing for Faculty membership. To start your RPS Faculty journey today, access the portfolio and tools at www.rpharms.com/Faculty

If your learning was planned in advance, please click:

If your learning was spontaneous, please click:

\section{References:}

[1] Asthma UK. Asthma facts and FAQs. 2015. Available at:_

https://www.asthma.org.uk/asthma-facts-and-statistics (accessed August 2018)

[2] Department of Health. An outcomes strategy for chronic obstructive pulmonary disease (COPD) and Asthma in England. Gateway reference: 16249. 2011. Available at:https://assets.publishing.service.gov.uk/government/uploads/system/uploads/attachment_data/file 1216139/dh_128428.pdf (accessed August 2018) 
[3] Royal College of Physicians. National review of asthma deaths: why asthma still kills? 2015. Available at: https://www.rcplondon.ac.uk/projects/outputs/why-asthma-stillkills (accessed August 2018)

[4] Department of Health. An outcomes strategy for chronic obstructive pulmonary disease (COPD) and Asthma in England: assessment of the impact on equalities (AIE). 2011. Available at:

https://assets.publishing.service.gov.uk/government/uploads/system/uploads/attachment_data/file 1216140/dh_128427.pdf (accessed August 2018)

[5] National Institute for Health and Care Excellence. Asthma. Quality standard [QS25]. 2017. Available at: https://www.nice.org.uk/guidance/QS25 (accessed August 2018)

[6] Mault S, McDonough B, Currie P \& Burhan H. P278 Reasons proffered for nonattendance at a difficult asthma clinic. Thorax 2012;67(Suppl 2):A187-A. doi:

$\underline{10.1136 / \text { thoraxjnl-2012- 202678.370 }}$

[7] van den Donk M, Sandbaek A, Borch-Johnsen K et al. Screening for type 2 diabetes. Lessons from the ADDITION-Europe study. Diabet Med 2011;28(11):14161424. doi: 10.1111/j.1464-5491.2011.03365.x

[8] Twigg MJ, Poland F, Bhattacharya D et al. The current and future roles of community pharmacists: views and experiences of patients with type 2 diabetes. Res Social Adm Pharm 2013;9(6):777-789. doi: 10.1016/j.sapharm.2012.10.004

[9] Beauchesne MF, Bercier D, Julien-Baker F et al. Community pharmacy-based medication assessment program for asthma and chronic obstructive pulmonary disease. Canadian Pharm J 2012;145(2):70-71. doi: 10.3821/145.2.cpj70

[10] Armour CL, Lemay K, Saini B et al. Using the community pharmacy to identify patients at risk of poor asthma control and factors which contribute to this poor control. $J$ Asthma 2011;48(9):914-922. doi: 10.3109/02770903.2011.615431

[11] Portlock J, Holden M \& Patel S. A community pharmacy asthma MUR project in Hampshire and the Isle of Wight. Pharm J 2009;282:109-112.

[12] Barbanel D, Eldridge S \& Griffiths C. Can a self-management programme delivered by a community pharmacist improve asthma control? A randomised trial. Thorax 2003;58(10):851-854. doi: 10.1136/thorax.58.10.851

[13] Wright D, Twigg M, Barton $\mathrm{G}$ et al. An evaluation of a multi-site community pharmacy- based chronic obstructive pulmonary disease support service. Int J Pharm Pract 2015;23(1):36-43. doi: 10.1111/ijpp.12165

[14] Wright D, Twigg M \& Thornley T. Chronic obstructive pulmonary disease case finding by community pharmacists: a potential cost-effective public health intervention. Int J Pharm Pract 2015;23(1):83-85. doi: 10.1111/ijpp.12161

[15] Fathima M, Naik-Panvelkar P, Saini B et al. The role of community pharmacists in screening and subsequent management of chronic respiratory diseases: a systematic review. Pharm Pract 2013;11(4):228-245. doi: 10.4321/S1886-36552013000400008

[16] Manfrin A, Thomas T \& Krska J. Randomised evaluation of the Italian medicines use review provided by community pharmacists using asthma as a model (RE I-MUR). BMC Health Services Res 2015;15:171. doi: 10.1186/s12913-015-0791-6

[17] Latif A, Pollock K \& Boardman HF. Medicines use reviews: a potential resource or lost opportunity for general practice? BMC Family Pract 2013;14:57. doi: 10.1186/1471-2296-14$\underline{57}$ 
[18] Murray R. Community Pharmacy Clinical Services Review. 2016. Available at: https://www.england.nhs.uk/commissioning/wp-content/uploads/sites/12/2016/12/communitypharm-clncl-serv-rev.pdf (accessed August 2018)

[19] British Medical Association, NHS Employers, NHS England. General Medical Services (GMS) contract Quality and Outcomes Framework (QOF): Guidance for GMS contract 2016/17. 2016. Available at:

http://www.nhsemployers.org/ /media/Employers/Documents/Primary\%20care\%20contracts/Q OF/2016-17/2016-17\%20QOF\%20guidance\%20documents.pdf (accessed August 2018)

[20] Garcia-Cardenas V, Armour C, Benrimoj SI et al. Pharmacists' interventions on clinical asthma outcomes: a systematic review. Eur Resp J 2016;47(4):1134-1143. doi: $\underline{10.1183 / 13993003.01497-2015}$

[21] NHS England. Clinical pharmacists in general practice. 2018. Available at: https://www.england.nhs.uk/gp/gpfv/workforce/building-the-general-practice-workforce/cp$\mathrm{gp} /$ (accessed August 2018)

[22] Craig P, Dieppe P, Macintyre S et al. Developing and evaluating complex interventions: the new Medical Research Council guidance. Int J Nurs Stud 2013;50(5):587592. doi: 10.1016/j.ijnurstu.2012.09.010

[23] Murphy A. Make asthma SIMPLE for your patients. Pharm J 2014;292(7809/10):515. doi: 10.1211/PJ.2014.11138140

[24] Nathan R, Sorkness C, Kosinski M et al. Development of the asthma control test: a survey for assessing asthma control. J Allergy Clin Immunol 2004;113:59-65. doi:

10.1016/j.jaci.2003.09.008

Citation: Clinical Pharmacist, August 2018, Vol 10, No 8, online | DOI:

10.1211/CP.2018.20205089 